Original Article

\title{
Characteristics and rehabilitation for patients with spinal cord stab injury
}

\author{
Fangyong Wang ${ }^{1,2)}$, Junwei Zhang ${ }^{1,2)}$, Hehu Tang ${ }^{1,2)}$, Xiang Li ${ }^{1,2)}$, Shudong Jiang ${ }^{1,2)}$, \\ Zhen Lv ${ }^{1,2)}$, Shujia Liu ${ }^{1,2)}$, Shizheng Chen $^{1,2)}$, Jiesheng Liu ${ }^{1,2)}$, Yi Hong $\left.{ }^{1,2}\right)^{*}$ \\ 1) Department of Spine and Spinal Cord Injury, Beijing Bo'ai Hospital, China Rehabilitation Research \\ Center, China \\ 2) School of Rehabilitation Medicine, Capital Medical University: Jiaomen North Road 10, Fengtai \\ District, Beijing 100068, China
}

\begin{abstract}
Purpose] The objective of the study was to compare the incidence, diagnosis, treatment, and prognosis of patients with spinal cord stab injury to those with the more common spinal cord contusion injury. [Subjects] Of patients hospitalized in China Rehabilitation Research Center from 1994 to 2014, 40 of those having a spinal cord stab injury and 50 with spinal cord contusion were selected. [Methods] The data of all patients were analyzed retrospectively. The cases were evaluated by collecting admission and discharge ASIA (American Spinal Injury Association) and ADL (activity of daily living) scores. [Results] After a comprehensive rehabilitation program, ASIA and ADL scores of patients having both spinal cord stab injury and spinal cord contusion significantly increase. However, the increases were noted to be higher in patients having a spinal cord stab injury than those having spinal cord contusion. [Conclusion] Comprehensive rehabilitation is effective both for patients having spinal cord stab injury and those with spinal cord contusion injury. However, the prognosis of patients having spinal cord stab injury is better than that of patients with spinal cord contusion.
\end{abstract}

Key words: Spinal cord injury, Stab injury, Rehabilitation

(This article was submitted Jul. 13, 2015, and was accepted Sep. 2, 2015)

\section{INTRODUCTION}

Spinal cord stab injuries are less frequently seen in clinical practice than spinal cord contusions. Younger age groups in certain countries are more likely to suffer from spinal cord stab injury because of the higher incidence of violence ${ }^{1)}$. Outside of those areas, however, spinal cord stab injury is uncommon.

From June 1994 to June 2014, 3,884 patients with spinal cord injury were treated at China Rehabilitation Research Center, 40 of whom had spinal cord stab injuries. The clinical data of 40 patients with spinal cord stab injury and 50 with spinal contusion injury, receiving the same treatment were analyzed and compared.

\section{SUBJECTS AND METHODS}

In the spinal cord stab injury group, there were 36 males and 4 females; 12 patients had complete injury to the spinal cord, and 28 had incomplete injury. Of the patients having incomplete spinal cord injury, 20 were diagnosed with

*Corresponding author. Yi Hong (E-mail: logan201410@163. com)

C2015 The Society of Physical Therapy Science. Published by IPEC Inc. This is an open-access article distributed under the terms of the Creative Commons Attribution Non-Commercial No Derivatives (by-ncnd) License $<$ http://creativecommons.org/licenses/by-nc-nd/3.0/>.
Brown-Séquard syndrome which is an incomplete spinal cord lesion characterized by a clinical picture reflecting hemisection injury of the spinal cord.

In the group of patients having spinal cord contusion injury, there were 45 males and 5 females; 17 patients with complete injury to the spinal cord, and 33 with incomplete injury. Of the patients with incomplete spinal cord injury, only 2 patients exhibited Brown-Séquard syndrome. The full demographic and clinical data of both groups are summarized in Table 1. The ethical committee of China Rehabilitation Research Center approved the studies, and written informed consent was obtained from each patient.

In acute stage of recovery, debridement of soft tissue was performed on all patients. In the group having spinal cord stab injury, 18 patients underwent operations such as spinal canal exploration, evacuation of hematoma, and removal of penetrating knives. In the group of patients with spinal cord contusion injury, all of the patients received routine operations of spinal canal decompression and internal fixation. In the chronic stage of recovery, patients in both groups were prescribed a comprehensive rehabilitation program including physical therapy (PT), occupational therapy (OT), acupuncture, and massage.

Evaluation scales $\left.{ }^{2}\right)$ utilized in rehabilitation settings to evaluate patient progress include the ASIA score for neurological function, and the FIM (Functional Independence Measure) score for activities of daily living (ADL). The ASIA and FIM scores were collected in both groups of patients upon admission and discharge. 
Paired t-test analysis was used to compare admission and discharge ASIA and ADL scores of patients having spinal cord stab injury to those of patients having contusion injury. Independent t-test analysis was used to compare ASIA and ADL score changes between the two groups.

\section{RESULTS}

After comprehensive rehabilitation, ASIA motor scores in patients having spinal cord stab injury increased from $58.03 \pm 20.54$ at admission to $69.13 \pm 17.93$ upon discharge $(p=0.014)$. ASIA sensory scores of the group with spinal cord stab injury increased from $146.21 \pm 41.68$ at admission to $166.11 \pm 34.22$ upon discharge $(\mathrm{p}=0.026)$. ADL scores of the group having spinal cord stab injury increased from $52.21 \pm 8.91$ at admission to $77.61 \pm 12.96$ on discharge $(p$ $=0.000$ ).

In the group of patients having spinal cord contusion injury, ASIA motor scores increased from $58.55 \pm 16.63$ at admission to $62.78 \pm 22.65$ upon discharge $(\mathrm{p}=0.121)$. ASIA sensory scores increased from $145.69 \pm 41.68$ at admission to $159.98 \pm 33.54$ upon discharge $(\mathrm{p}=0.035)$. ADL scores increased from $52.73 \pm 12.76$ at admission to $64.83 \pm$ 9.60 upon discharge $(\mathrm{p}=0.017)$.

The ASIA motor score increase in patients having spinal cord stab injury and those having spinal cord contusion injury was $11.14 \pm 3.78$ and $4.13 \pm 1.67$, respectively $(p=$ 0.022). The ASIA sensory score increase in patients having spinal cord stab injury and those having spinal cord contusion was $20.45 \pm 4.66$ and $14.43 \pm 2.68$, respectively $(\mathrm{p}=$

Table 1. Demographic clinical data from spinal cord stab injury and contusion injury groups

\begin{tabular}{llcc}
\hline Items & Sub-items & $\begin{array}{c}\text { Stab injury } \\
\text { group }\end{array}$ & $\begin{array}{c}\text { Contusion } \\
\text { injury group }\end{array}$ \\
\hline Gender & $\mathrm{M}$ & 36 & 45 \\
& $\mathrm{~F}$ & 4 & 5 \\
\multicolumn{2}{l}{ Age (years) } & $24.3(18-45)$ & $28.4(22-65)$ \\
IL & Cervical level & 5 & 10 \\
& Thoracic and lumbar level & 35 & 40 \\
ID & Incomplete injury & 28 & 33 \\
& Complete injury & 12 & 17 \\
BSS & & 20 & 2 \\
\hline
\end{tabular}

F: Female; M: Male; IL: Injury level; ID: Injury degree; BSS: Brown-Séquard syndrome
0.031). The ADL score increase in the group having spinal cord stab injury was $25.87 \pm 9.82$, compared with $12.55 \pm$ 6.43 in the group with contusion injury $(p=0.005)$. The follow-up results of both groups on admission and discharge are summarized in Tables 2-4.

\section{DISCUSSION}

Spinal cord stab injury is an open injury of the spinal cord by sharp objects whose pathological change, clinical manifestation, and prognosis differ from those of other kind spinal cord injury. In the past, large research samples of patients having a spinal cord stab injury were seldom reported in the literature, but the incidence is increasing in recent years ${ }^{3)}$. According to Karacan I et al. ${ }^{1)}$, youngsters are more likely to suffer from spinal cord stab injury, which is similar with our study in which the mean age of spinal cord stab injury patients is 24.3 years old.

Most spinal cord stab injuries occur at the thoracic and lumbar levels, only in a few cases they occur at the cervical level. This is because of more segments and increased vertebral height of the thoracic and lumbar vertebral levels, making them more likely to be injured by sharp objects. As a result of the special injury mechanism, there are more

Table 2. Admission and discharge ASIA and ADL scores of 40 patients with spinal cord stab injury

\begin{tabular}{lccc}
\hline Items & On admission & On discharge & p-value \\
\hline ASIA motor score & $58.0 \pm 20.5$ & $69.1 \pm 17.9$ & $*$ \\
ASIA sensory score & $146.2 \pm 41.7$ & $166.1 \pm 34.2$ & $*$ \\
ADL score & $52.2 \pm 8.9$ & $77.6 \pm 13.0$ & $*$ \\
\hline
\end{tabular}

ASIA: American Spinal Injury Association; ADL: Activity of daily living

$* \mathrm{p}<0.05$

Table 3. Admission and discharge ASIA and ADL scores of 50 patients with spinal cord contusion injury

\begin{tabular}{lccc}
\hline Items & On admission & On discharge & p-value \\
\hline ASIA motor score & $58.6 \pm 16.6$ & $62.8 \pm 22.7$ & \\
ASIA sensory score & $145.7 \pm 41.7$ & $160.0 \pm 33.5$ & $*$ \\
ADL score & $52.7 \pm 12.8$ & $64.8 \pm 9.6$ & $*$ \\
\hline
\end{tabular}

ASIA: American Spinal Injury Association; ADL: Activity of daily living

$* \mathrm{p}<0.05$

Table 4. ASIA and ADL score improvement from admission to discharge of patients with spinal cord stab and contusion injury

\begin{tabular}{lccc}
\hline Items & $\begin{array}{c}\text { Stab injury } \\
\text { group }\end{array}$ & $\begin{array}{c}\text { Contusion injury } \\
\text { group }\end{array}$ & \\
\hline ASIA motor score increase & $11.1 \pm 3.8$ & $4.1 \pm 1.7$ & $*$ \\
ASIA sensory score increase & $20.5 \pm 4.7$ & $14.4 \pm 2.7$ & $*$ \\
ADL score increase & $25.9 \pm 9.9$ & $12.6 \pm 6.4$ & $*$ \\
\hline
\end{tabular}

ASIA: American Spinal Injury Association; ADL: Activity of daily living ${ }^{*} \mathrm{p}<0.05$ 
incomplete cord injuries occurring after stab injury than contusion injuries. The midline spinal process prevents sharp objects from crossing midline before entering the ligamentum flavum, leading to hemi-section of the spinal cord. Ipsilateral fasciculus gracilis and fasciculus cuneatus are the first to be injured. Lateral corticospinal and spinothalamic tracts are the second to be injured. Once sharp objects reach the anterior spinal cord, they may cross midline and cut anterior column tissue. When being penetrated the spinal column is usually in the position of flexion. Sharp objects penetrate the spinal canal between spinal processes leading to dura rupture and bleeding of the spinal cord. Sometimes, a hematoma will compress the spinal cord, causing complete or incomplete injury. Clinically, more than half of spinal cord stab injuries manifest as Brown-Séquard syndrome ${ }^{4)}$. In our study, $46.7 \%$ of the patients exhibited Brown-Séquard syndrome.

Spinal cord stab injury areas are not large compared to those of contusion, in which secondary spinal cord injury is very severe. However, the main cause of paraplegia following a stab wound is the primary injury of the spinal cord. Patients in the spinal cord stab injury group exhibit good functional recovery, and even change from complete to incomplete injury.

The first treatment for spinal cord stab injury is to change the open injury to a closed injury. Most spinal cord stab injuries do not influence spinal stability, so fixation is not necessary. Spinal cord MRI is a good option for diagnosing spinal cord stab injuries ${ }^{5}$. Spinal cord stab injuries having evidence of a knife inside the canal require surgery, including exploration of the spinal canal, hematoma evacuation, and removal of the intervertebral disk and/or bone fragments. The purpose of surgery is also to prevent infection and stop severe bleeding in the spinal canal, both of which will contribute to recovery. Once leakage of cerebrospinal fluid occurs, dura suturing should be performed immediately. Spinal cord stab injuries have a higher probability of developing an epidural hematoma than spinal cord contusion injuries. Methylprednisolone (MP) injection can protect the spinal cord from secondary harm and provide conditions for optimal recovery following injury. However, whether MP should be used following spinal cord injury needs further study. Just as a comprehensive rehabilitation program including physical therapy (PT), occupational therapy (OT), acupuncture, and massage, etc. is helpful for other patients with spinal cord injury6), it is also very important for those having spinal cord stab injuries. The patient's rehabilitation program should be initiated as soon as possible because it will significantly facilitate their neurological recovery, improve their activities of daily living, shorten their hospital stay, and reduce their overall medical costs.

In conclusion, comprehensive rehabilitation programs are effective for both patients having spinal cord stab and contusion injuries. The prognosis of patients having spinal cord stab injuries is better than of those having spinal cord contusion.

\section{ACKNOWLEDGEMENT}

Grant support for this study was provided by Capital Medical Development Fund of Beijing, China (No. 20144-4144).

\section{REFERENCES}

1) Karacan I, Koyuncu H, Pekel O, et al.: Traumatic spinal cord injuries in Turkey: a nation-wide epidemiological study. Spinal Cord, 2000, 38: 697701. [Medline] [CrossRef]

2) Kirshblum SC, Waring W, Biering-Sorensen F, et al.: Reference for the 2011 revision of the International Standards for Neurological Classification of Spinal Cord Injury. J Spinal Cord Med, 2011, 34: 547-554. [Medline] [CrossRef]

3) Saeidiborojeni HR, Moradinazar M, Saeidiborojeni S, et al.: A survey on spinal cord injuries resulting from stabbings: a case series study of 12 years' experience. J Inj Violence Res, 2013, 5: 70-74. [Medline]

4) Pascual MG, Alcácer VS, Pomares MV, et al.: Brown-Séquard-plus syndrome after a stab injury. NeuroRehabilitation, 2011, 29: 353-357. [Medline]

5) Emich S, Weymayr F, Steinbacher J, et al.: The role of MRI in spinal stab wounds compared with intraoperative findings. Eur Spine J, 2012, 21: S535-S541. [Medline] [CrossRef]

6) Anwer S, Equebal A, Palekar TJ, et al.: Effect of locomotor training on motor recovery and walking ability in patients with incomplete spinal cord injury: a case series. J Phys Ther Sci, 2014, 26: 951-953. [Medline] [CrossRef] 